IILLNESS, CRISIS \& LOSS, Vol. 22(2) 115-126, 2014

\title{
NORMALCY: AN IMPORTANT FOCUS FOR INDIVIDUALS DIAGNOSED WITH A HAEMATOLOGICAL MALIGNANCY
}

PAM MCGRATH, BSOCWK, MA, PH.D.

Griffith University, Queensland, Australia

\begin{abstract}
This article presents findings that highlight the significance of the tension between the focus on disease and treatment verses the focus on re-engaging with life for individuals facing the crisis of being diagnosed with a haematology malignancy. The findings are from a study on survivorship conducted throughout Queensland, Australia. The research was based on a qualitative design involving a series of open-ended interviews $(n=50)$ and one focus group which were recorded, transcribed verbatim, and then coded and thematically analysed for the research. The findings affirm the importance of a sense of normalcy and the process of re-positioning the central role of the disease in the lives of those diagnosed with a haematological cancer. There are key points when a focus on treatment and disease is unavoidable. However, after treatment the need for supportive care and the consequent reminder of the disease and treatment can "taper off." The challenge is to know when to provide support and when to respect the individual's need to let go of a focus on issues associated with treatment and the disease.
\end{abstract}

Key Words: haematology, supportive care, normalcy, qualitative research

(c) 2014, Baywood Publishing Co., Inc. doi: http://dx.doi.org/10.2190/IL.22.2.c http://baywood.com 
As an outcome of advances in treatment, many haematological cancers that were once quickly fatal are now considered "chronic" or long-term medical conditions (Maher \& De Vries, 2011; McGrath, 2008). The curative success with paediatric acute lymphoblastic leukaemia and Hodgkin's lymphoma are legendary. However, in recent years, other conditions such as myeloma, chronic myeloid leukaemia, and some non-Hodgkin's lymphomas are also showing significant improvements in rates of longevity and survival (Anstrom, Reed, Allen, Glendenning \& Schulman, 2004; Kyle \& Rajkumar, 2004; Van Oers, Klasa, \& Marcus, 2006). By way of example, myeloma is now referred to as an "incurable chronic disease" (Maher \& De Vries, 2011). Such success is to be celebrated as it has shifted the perspective for many haematological conditions from acute to chronic, with many individuals in these diagnostic groups now able to re-engage with life and the future.

In recent decades, the focus in cancer care has broadened to include a psychosocial perspective that involves a move from a predominantly clinical focus to include a holistic understanding of the patient and their families (Holland \& Weiss, 2008). Indeed, there are now calls to ensure that all cancer patients receive adequate rehabilitation and long-term support following episodes of acute cancer care (Doyle \& Kelly, 2005; Molassiotis, Wilson, Blair, Howe, \& Cavet, 2011). The assumption is made, without evidence, that individuals diagnosed with cancer will want ongoing support and are accepting of being identified as needing long-term follow-up. As a consequence, there are now a range of supportive care initiatives designed for cancer survivors (Galantino, Schmid, Botis, Dagan, Leonard, \& Milos, 2010). This assumption of the need for the provision of ongoing support for cancer patients is made despite the fact, as Doyle and Kelly note (2005), that there is a lack of available research evidence to guide the long-term support of cancer patients.

The findings presented in this article, from a major survivorship study for individuals diagnosed with a haematological malignancy conducted throughout Queensland, Australia, challenge the very notion of the appropriateness of the need for ongoing supportive care involvement for all patients diagnosed with cancer. While acknowledging that receiving a diagnosis of a haematological malignancy is a crisis for any individual, it does not necessarily follow ipso facto that a continuing focus on the illness is a necessary or positive strategy. The article describes the important role of normalcy and the consequent tension between a focus on disease and treatment verses a focus on re-engaging with life for individuals diagnosed with haematology. Although of great relevance to supportive care planning and delivery, the role of normalcy has, as yet, received scant attention in the research literature. This article describes issues associated with the focus on normalcy and the tension generated with an ongoing focus on disease from the perspective of individuals diagnosed with a haematological cancer. The findings are set in the context of the implications for supportive care service providers. 


\section{THE RESEARCH}

The research project was funded by the Leukaemia Foundation of Queensland (LFQ). The aim of the research was to document and explore issues associated with the experience of survivorship for haematology patients supported by LFQ. Overall, the study provides practical insights to inform supportive care service delivery with regards to effectively engaging with and supporting individuals coping with a range of psycho-social issues associated with a haematology malignancy (McGrath \& Holewa, 2011).

A qualitative design (Holloway, 2008; Patton, 2002) based on a series of open-ended interviews and one focus group was utilised to explore and document the experience of survivorship from the perspective of adult patients diagnosed with a haematological malignancy. For the purpose of this research, a "survivor" was defined as an adult individual with a haematological malignancy who was at least 1 year post-diagnosis. This definition was established in collaboration with LFQ and resonates with Feuerstein's (2007) definition of the term as the period from completion of primary treatment up to end-of-life care. The focus group was used as a forum to discuss the findings with a separate group of haematology survivors and to affirm or extend the conclusions reached.

\section{PURPOSIVE SAMPLE OF PARTICIPANTS}

Participants were purposively sampled from a database of patients maintained by LFQ. The participants were enrolled by two Project Officers who were under contract with CQUniversity and independent of LFQ. Potential participants received a letter from LFQ that informed them of the study and stated that if the person did not want to participate they could contact LFQ to opt out. Any individual not wanting to be involved in the research was deleted from the list. At this stage, the database of patient contacts, excluding the details of those who chose to withdraw from the study, was provided to the Project Officers for participant selection. Thus, the actual identity of those who did participate remained confidential, as LFQ was not provided with any details of the actual selection. Potential participants were provided with a written Project Description and Consent Form and an initial telephone call inviting participation in the research. Prior to interviewing, participants were again informed of their ethical rights (e.g., informed consent, confidentiality, right to withdraw) and individual consent was obtained. The CQUniversity Human Research Ethics Committee approved the study.

All participants had to meet the criteria for survivorship in that they were an adult individual with a haematological malignancy who was at least 1 year post-diagnosis. One hundred and eighteen potential participants were contacted with 14 declining to participate and 54 being un-contactable (due to a change in contact details). In total, there were 50 participants ( $n=26$ male; $n=24$ female) 
which represented the major haematological diagnostic groups: Multiple Myeloma $(n=15)$, Lymphoma $(n=14)$, Leukaemia $(n=17)$, and Other $(n=4)$. Of the overall cohort, 11 participants had a Bone Marrow Transplant and 15 had a Stem Cell Transplant (allogeneic and autologous transplants). Due to the unique geography, population, and service provision patterns of Queensland, Australia, a customised regional classification system was designed and used to ensure that the purposive sample included participants that had varying levels of access to haematological services based upon their home address. The sample also ensured a representation of ages across the adult life span. At the completion of data collection, the findings were provided to a group of seven focus group participants for comment. Project management and collaboration was managed using the online qualitative collaboration software, Quadrant (www.quadrant.com).

\section{INTERVIEWS AND FOCUS GROUP}

The exploration of the experience of survivorship from the consumers' perspective was conducted through an iterative, qualitative research methodology using open-ended telephone interviews at the time and location chosen by each participant. The interviews began with an invitation to the participant to talk about their experience since diagnosis and treatment for a haematological malignancy. A list of topic areas to explore during the interviews was developed from consultations with LFQ, published research, and anecdotal comments. However, in accordance with the iterative principle of qualitative research, the issues being explored evolved with the study, with early insights informing the discussion in subsequent interviews. In relation to the sub-set of findings presented in this article, all participants were asked to talk about their experience, thoughts, and attitudes toward a range of supportive care initiatives provided by LFQ. The interviews proceeded at the pace and direction of the interviewee and included techniques of probing, clarification, paraphrasing, and summarising to explore each participant's experience (Seidman, 2006). The interviews were conducted by the primary investigator who has a background as a haematology practitioner with extensive experience in conducting qualitative research in haematology, and the trained project officer who has also worked for many years in qualitative haematology research. Both interviewers are skilled in open-ended, Rogerian style interviewing and have a well-established process of reviewing the progress of the interviews to ensure active-listening to the voice of the participant. The interviews lasted for approximately 1 hour and were audio-recorded and transcribed verbatim. The focus group participants were presented with a PowerPoint summary of the findings from the interviews, and were encouraged to comment by expressing agreement or disagreement and providing further thoughts on the issues. The focus group discussion was recorded and transcribed verbatim and the findings affirmed the individual participant findings. 
NORMALCY WITH HAEMATOLOGICAL MALIGNANCY / 119

\section{Analysis}

After transcription, the language texts were entered into the qualitative data analysis computer program NVivo and analysed thematically. All of the participants' comments were coded into "free nodes," which are category files that have been "freely" created from the data rather than pre-organised. The list of codes were then transported to Word (Word 2007) and organized under thematic headings. The coding was established by an experienced qualitative researcher and completed by a Project Officer with extensive experience in coding qualitative data. There was agreement between researcher and coder on the coding and emergent themes created from the transcriptions.

Further findings from the study based on the same methodology are starting to be published, such as articles on the concept of "chemo brain," return-to-work issues, sexuality, and the concept of survivorship (McGrath, 2012; McGrath, Hartigan, Holewa, \& Skaparis, 2011a, 2011b; McGrath \& Holewa, 2012).

The information in the two codes named "maintaining normalcy" and "provision of support" form the basis for the findings presented in this article. Identifiers used in the Results section are age, gender (F-female; $\mathrm{M}$-male), diagnosis (APML-Acute Promyelocytic Leukaemia; ALL-Acute Lymphoblastic Leukaemia; AML-Acute Myeloid Leukaemia; CML-Chronic Myeloid Leukaemia; NHL-Non-Hodgkin's Lymphoma; MM-Multiple Myeloma); and whether they have undergone a transplant (BMT-Bone Marrow Transplant; SCT-Stem Cell Transplant).

\section{FINDINGS}

\section{The Tension between a Focus on Living and a Focus on Disease and Treatment}

Participants made it clear that post-treatment their desire was to focus on life and not on the disease and treatment. For example:

See I don't really want to know too much about it (disease and treatment) much more. I just want to get on with living and if it comes back I know I'm in big trouble but no, I'm happy the way things are there. (NHL_F_65yrs_SCTrans)

Because we want to focus on the living rather than on the condition ... yeah, we've put that behind us and we're just going forward. (MM_M_68yrs_SCTrans)

The focus on living rather than illness and treatment does not deny the cancer but rather involves an acceptance and re-positioning of the central role of the disease, for example, 
Oh well it's cancer, it's incurable but it is treatable. If it comes back again I'll just go and have another fix and it's not going to go away, nothing's going to make it go away so you may as well get on with it (life). (NHL_F_70yrs)

Yeah try to get on with life and not let it (disease) run your life. (CML_M_53yrs)

The participants reported a tension between the focus on disease and treatment and their desire to maintain their focus on living:

... I find myself wavering between the two. . . . I'm aware of that I waver; there are times when I just blank it out completely and I don't want to be reminded about it and there are times when I think yes but if you, it made you who you are now. ... (APML_F_40yrs)

There was a belief that to some degree the individual can choose a predominant focus on the disease or re-engagement with life, for example,

I think you can put it (focus on disease) away or dwell on it.

(ALL_M_66yrs_BMT)

However, even if choosing a predominant focus on life there are unavoidable times when the individual is reminded of the disease and treatment, for example,

The time I focus on my condition is when I take my medication in the morning, when I take it in the night and if I suddenly feel a temperature and that's about the only time I have on it in reality. (MM_M_63yrs_SCTrans)

\section{The Focus is Not Fixed but Changes Over Time}

The competing tension between focusing on the disease versus focusing on life is a fluid, continuing tension that evolves and changes over time depending on the circumstances of the individual, for example:

Yeah probably a little bit of both, sometimes it'd be nice (to talk about the cancer) and other times you're just like, "aw I'm through that now," like I don't know, you don't want to look back kind of thing. (ALL_F_21yrs)

The participants identified a number of points in time when there was a strong need for support in relation to the disease and treatment which included:

- at diagnosis;

- beginning treatment;

- initially after leaving hospital post-treatment;

- before undergoing a transplant (bone marrow or peripheral stem cell transplant); and

- during relapse.

An example of the change over time from the treatment period to post-treatment is well explained by the following statement: 
Yep well I guess for me back when I was having the treatment there was no, there was no way of avoiding not thinking about, avoid thinking about it because it was your whole life. It affected every minute of every day you know of every week of every year for those couple of years. So I think it had to become a sole focus for a little while there ... now it gets to the point where, "that's right I had leukaemia" (laughs), that's a really good point to be at you know? It's only every now and then I kind of think, "aw yeah." You know obviously I still have all the memories of the bad times but on the day-to-day basis it's not invading my life in a big way. (APML_F_40yrs)

Even for those with a strong need to talk to others about the diagnosis and treatment, the need to discuss these topics can taper off over time:

But you know, the need's got less to do that (talk about diagnosis and treatment) over the years. (MM_F_70yrs)

As an indication of the changes in need over time, many of the participants indicated that at the time of the interview there was no need for support with regards to their condition or treatment:

That might've been four or five years ago, it's not something at the forefront of my mind at the moment. (CML_M_50yrs_BMT)

\section{The Challenge for Supportive Care}

Individuals spoke of the process of "moving on" after the intense period associated with diagnosis and treatments have ended, for example:

I pretty much moved on. I don't really feel an urge to. I always remember and stuff what they've (LFQ) done for me but I don't really think I need to keep in touch. (ALL_F_22yrs_BMT)

The focus moves from the cancer and treatment to the process of getting on with living:

. . . but they often send me letters saying there's a talk here or a talk there but they're all in Brisbane you know and again I don't, I don't see anything that I'd learn from it. Yeah, I want to get on with life, I don't want it thrown up [in] my face you know every so often. Going down to any meeting's not going to help me, you know it may help other people I don't know, but it's not going to help me. (ALL_M_66yrs_BMT)

For these individuals, there was an acknowledgment with gratitude of the help that the Leukaemia Foundation of Queensland previously provided juxtaposed against a sense of present independent coping, for example:

Well I feel they've done us, I feel they've done very well. I feel that there's not much else really they can do, especially in my position where I'm working. If I was confined to bed or that sort of thing that'd be different you know, probably be looking for some help like that but the way we're 
going at the moment up to the present time I feel that I you know, we're managing quite well. (MM_M_65yrs)

The challenge for supportive care professionals is to know when to connect and support and when to provide individuals with their personal space (and time out from focusing on the disease) to get on with life. The balance is important to survivors, as can be seen by the following statement:

I just need to move away from it all, it's overwhelming me right now, I just want to be a normal person and they (supportive care organisation) went "okay, yeah no that's fine." It's not that they've not done their job, it's not that I don't need them anymore, it's just that I need space just right now and it varies. (APML_F_40yrs)

The important supportive care consideration is that even though there are times when individuals want the space to get on with living and focus on re-engaging with life and not want to be directly involved in support activities, they may still want to retain the connection with the supportive care organisation. As clearly stated by the following participant, the challenge for supportive care is to maintain the life-line so people can return when the need arises:

... but the thing is it's not relevant anymore to me... . I've sort of moved on and gone beyond that so it's not as if I'm still suffering from the same thing, I'm suffering from something which has been caused by that but it's now entirely different. ... I don't want to lose contact with them. . . I don't want them sort of ringing me up all the time either. (AML_M_50yrs_BMT)

\section{DISCUSSION}

There is increasing awareness in the literature of the cancer patient's desire to re-engage with a sense of normalcy in their life after treatment. However, most of the discussion on this topic is tangential to other findings on survivorship and based on research with diagnostic groups, mainly breast cancer, that were not inclusive of haematological malignancies (DeMarco, Picard, \& Agretelis, 2004; Travis, 2011; Walker, Nail, Larsen, Magill, \& Schwartz, 1996). Mather and De Vries's (2011) research notes the ever-shifting perspective between illness and wellness for myeloma patients but relates this to a sense of uncertainty. The important point is that, to date, the issue of normalcy has not received the significant focus it requires. Although mentioned, it has only been dealt with as a minor issue and certainly not prioritised in relation to post-diagnosis care of haematology patients.

The findings presented in this article affirm the importance of a sense of normalcy for those diagnosed with a haematological cancer. Keeping the focus on living rather than on illness and treatment does not deny the cancer but rather involves an acceptance and re-positioning of the central role of the disease. 
An understanding of the importance of maintaining a sense of normalcy, however, is only part of the story.

The focus on living was reported by the participants in the study to be an important coping strategy that can be consciously chosen by the individual. However, the ability to keep the focus away from the disease and treatment changes over time. There are key points in the individual's journey with a haematological malignancy when a focus on treatment and disease can be unavoidable, including at diagnosis, the beginning of treatment, immediately after leaving hospital post-treatment, before transplant, and during relapse. It is at these points in time when individuals can be most in need of and receptive to support offered by oncology supportive care service providers. However, after treatment the need for such care and the consequent reminder of the disease and treatment can "taper off." The individuals interviewed for the study reported a sense of "moving on": a positive feeling of coping independently with a focus on re-engaging with normalcy (albeit newly defined), life, and their future.

The challenge for oncology supportive care professionals is to know when to provide support and when to respect the individual's need to let go of a focus on issues associated with treatment and the disease. Many supportive care organisations and services are directly indentified with the disease and treatment through their association with hospitals, clinics, or cancer societies. In the case of the LFQ, the very name of the organisation is a reminder of the disease and treatment. However, the findings are not a case against the naming of supportive care in diagnostic terms as there is an obvious role for this. But rather, the findings indicate that consideration needs to be given to the impact that such identification means to individuals diagnosed with haematological malignancies. The times when individuals want to "move on" from supportive care are to be viewed as a positive and normalised coping strategy, not as a failure of supportive care or a form of denial in the patient. By way of example, attrition from a support group may be a result of an individual wanting to "move on" in a positive way rather than a problem, per se, with the group itself. Indeed, the desire for individuals to re-engage with life and to cope independently can be an indication of positive growth from the support and care provided by the group (McGrath, 2003). In short, the findings challenge the present assumption in the literature (Doyle \& Kelly, 2005; Molassiotis et al., 2011) that all individuals diagnosed with a haematological malignancy will require long-term and consistent follow-up. The indications are that strategies are required to ensure that support is offered to those in need while at the same time respecting the privacy and life-focus of those who do not require the support.

Further findings from the study indicated that the establishment of a strong and caring relationship with the haematology patient and their family is the most effective strategy that LFQ uses to maintain this balance of connection. The supportive care professionals from LFQ provide direct personal, practical, and information support to haematology patients and their families during intense 
times of need. It is during these times of response to need that a meaningful relationship is built with the patient and their family. It is this relationship that provides the safety net for negotiating the shifting focus reported in this article. The relationship provides the life line that allows for moving on at times when the focus is predominantly on living while knowing that the foundation is there if or when the need arises.

\section{CONCLUSION}

The findings presented in this article posit an important notion for supportive care that, as yet, has not been adequately addressed by the literature. The idea that there needs to be a flexible approach to supportive care delivery that respects the ever-changing focus from treatment and disease to re-engaging with life needs to be further explored through research. The present research indicates that this is an important notion for haematology patients; however, further research is required to explore the idea with other cancer diagnostic groups. The hope and expectation is that a direct outcome of putting this notion on the research and supportive care agenda is that it will contribute to deepening our understanding of how best to respond across the many stages associated with the cancer journey.

\section{ACKNOWLEDGMENTS}

The author would like to thank the research team for their contribution to the study, including Mr. Hamish Holewa, Ms. Nicole Rawson-Huff, Ms. Bo McGrath, Mr. Michael Bouwman, Ms. Mary Anne Patton, and Mrs. Emma Phillips. The authors would also like to thank Mrs. Barbara Hartigan, Ms. Beverley Mirilo, and Mrs. Maryanne Skarparis from the Leukaemia Foundation of Queensland. The authors would like to thank CQUniversity, the institution in which the data was collected and Griffith University, the institution in which the findings were analysed and written up.

\section{REFERENCES}

Anstrom, K., Reed, S., Allen A., Glendenning, P., \& Schulman, K. (2004). Long-term survival for imatinib versus interferon-alpha plus low-dose cytarabine for patients with newly diagnosed chronic-phase chronic myeloid leukemia. Cancer, 101(11), 2584-2592.

De Marco, R., Picard, C., \& Agretelis, J. (2004). Nurse experiences as cancer survivors: Part I-personal. Oncology Nursing Forum, 31(3), 523-530.

Doyle, N., \& Kelly, D. (2005, September-December). "So what happens now?" Issues in cancer survival and rehabilitation. Clinical Effectiveness in Nursing, 9(3-4), 147-153. 
Feuerstein, M. (2007). Defining cancer survivorship. Journal of Cancer Survivorship, 1, 5-7.

Galantino, M., Schmid, P., Botis, S., Dagan, C., Leonard, S., \& Milos, A. (2010). Exploring wellness coaching and traditional group support for breast cancer survivors: A pilot study. Rehabilitation Oncology, 28(1), 19-25.

Holland, J., \& Weiss, T. (2008, November-December). The new standard of quality cancer care: Integrating the psychosocial aspects in routine cancer from diagnosis through survivorship. Cancer Journal, 14(6), 425-428.

Holloway, I. (2008). A-Z of qualitative research in healthcare. Oxford, UK: Blackwell.

Kyle, R., \& Rajkumar, V. (2004). Multiple myeloma. New England Journal of Medicine, $351,1860-1873$.

Maher, K., \& De Vries, K. (2011, March). An exploration of the lived experiences of individuals with relapsed multiple myeloma. European Journal of Cancer Care, 20(2), 267-275.

McGrath, P. (2003). "Living well with grief': Insights on bereavement support. AustralAsian Journal of Cancer, 2(3), 225-233.

McGrath, P. (2008). Living with leukaemia, lymphoma and myeloma: A guide for patients and their families. Trivandrum, Kerala, India: Researchman.

McGrath, P. (2012). The impact on sexuality after diagnosis and treatment for a haematological malignancy: Findings from Australia. Oncology Nursing Forum, 39(6), 595-600.

McGrath, P., Hartigan, B., Holewa, H., \& Skaparis, M. (2011a). "Chemo brain": Research findings indicate need for caution. Austral-Asian Journal of Cancer, 10(3), 33-41.

McGrath, P., Hartigan, B., Holewa, H., \& Skarparis, M. (2011b). Returning to work after treatment for haematological cancer: Findings from Australia. Supportive Care in Cancer, 20, 1957-1964.

McGrath, P., \& Holewa, H. (2007). Description of an Australian model for end-of-life care in patients with haematologic malignancies. Oncology Nursing Forum, 34(1), 79-85.

McGrath, P., \& Holewa, H. (2011). Leukaemia Foundation of Queensland Survivor Report. Brisbane, Australia.

McGrath, P., \& Holewa, H. (2012). What does the term "survivor" mean to individuals diagnosed with a haematological malignancy? Findings from Australia. Supportive Care in Cancer, 20, 3287-3295.

Molassiotis, A., Wilson, B., Blair, S., Howe, T., \& Cavet, J. (2011, January). Unmet supportive care needs, psychological well-being and quality of life in patients living with multiple myeloma and their partners. Psycho-Oncology, 20(1), 88-97.

Patton, M. (2002). Qualitative evaluation and research methods (2nd ed.). Newbury Park, CA: Sage.

Seidman, I. (2006). Interviewing as qualitative research: Guide for researchers in education and the social sciences (3rd ed.). New York, NY: Teachers College Press.

Travis, P. (2011). Presenteerism at the work place. Danish Journal of Nursing, 111(10), 48-1.

Van Oers, M., Klasa, R., \& Marcus, R. (2006). Rituximab maintenance improves clinical outcome of relapsed/resistant follicular non-Hodgkin lymphoma in patients 
126 / MCGRATH

both with and without rituximab during induction: Results of a prospective randomised phase 3 intergroup trail. Blood, 108(10), 3295-3301.

Walker, B., Nail, L., Larsen, L., Magill, J., \& Schwartz, A. (1996, September). Concerns, affect, and cognitive disruption following completion of radiation treatment for localized breast or prostate cancer. Oncology Nursing Forum, 23(8), 1181-1187.

\section{Direct reprint requests to:}

Pam McGrath

Associate Professor

P.O. Box 1307

Kenmore Q1d 4069

Australia

e-mail: pmcgrathgu@gmail.com orp.mcgrath@griffith.edu.au 